\title{
The microRNA expression changes associated with malignancy and SDHB mutation in pheochromocytoma
}

\author{
E Patterson, R Webb, A Weisbrod, B Bian, M He, L Zhang, A K Holloway ${ }^{1}$, \\ $R$ Krishna, N Nilubol, K Pacak ${ }^{2}$ and $E$ Kebebew
}

\author{
Endocrine Oncology Section, NIH/NCI/Surgery Branch, National Cancer Institute, NIH, Hatfield Clinical Research Center, Room \\ 4-5952, 10 Center Drive, Bethesda, Maryland 20892, USA \\ ${ }^{1}$ Gladstone Institutes, University of California, San Francisco, California 94158, USA \\ ${ }^{2}$ Program in Reproductive and Adult Endocrinology, Eunice Kennedy Shriver National Institute of Child Health and Human \\ Development (NICHD), NIH, Bethesda, Maryland 20892, USA
}

(Correspondence should be addressed to E Kebebew; Email: kebebewe@mail.nih.gov)

\begin{abstract}
Currently, the diagnosis of malignant pheochromocytoma can only be made when there is clinical evidence of metastasis or extensive local invasion. Thus, there is a need for new diagnostic marker(s) to identify tumors with malignant potential. The purpose of this study was to identify microRNAs (miRNAs) that are differentially expressed between benign and malignant pheochromocytomas and assess their diagnostic accuracy. Toward this aim, we analyzed miRNA expression in benign and malignant pheochromocytoma tumor samples using whole genome microarray profiling. Microarray analysis identified eight miRNAs that were significantly differentially expressed between benign and malignant pheochromocytomas. We measured a subset of these miRNAs directly by RT-PCR and found that miR-483-5p, miR-183, and miR-101 had significantly higher expression in malignant tumors as compared to their benign counterparts. Area under the receiver operating curve (AUC) analysis indicated that miR-483-5p, miR-101, and miR-183 could be useful diagnostic markers for distinguishing malignant from benign pheochromocytomas. In addition, these miRNAs could be detected in pheochromocytoma patient serum. Overall our data suggest that misexpression of miR-483-5p, miR-101, and miR-183 is associated with malignant pheochromocytoma.
\end{abstract}

Endocrine-Related Cancer (2012) 19 157-166

\section{Introduction}

Pheochromocytomas are neuroendocrine tumors that originate in the adrenal medulla or at extra-adrenal sites. Malignant pheochromocytoma is defined by the presence of tumor at a site where chromaffin cells are not normally located (Zarnegar et al. 2006). The prevalence of malignant pheochromocytoma is 2.5 $40 \%$, and the overall survival rate is $<50 \%$ at 5 years (Zarnegar et al. 2006). Currently, there are no reliable histologic or molecular markers for distinguishing between benign and malignant localized pheochromocytomas. Pheochromocytoma can be associated with autosomal hereditary cancer syndromes, such as multiple endocrine neoplasia type 2 (MEN2A and MEN2B), von Hippel-Lindau syndrome (VHL), paraganglioma syndrome type 1, 3, and 4 (PGL1, PGL3, and PGL4), and neurofibromatosis 1 (NF1). More recently, novel susceptibility genes such as TMEM127 and MAX have been identified in familial pheochromocytoma and PGL (Qin et al. 2010, Yao et al. 2010, Comino-Mendez et al. 2011, Neumann et al. 2011). Although germline mutation in genes including RET, VHL, SDHC, SDHB, SDHD, TMEM127, MAX, or NF1 is associated with developing pheochromocytoma/PGL, the molecular events involved in malignant transformation are poorly understood.

MicroRNAs (miRNAs) are short, noncoding RNAs that posttranscriptionally regulate gene expression by sequence-specific targeting of mRNAs. miRNAs have 
roles in diverse biological processes, such as differentiation, cellular growth, and apoptosis. Therefore, it is likely that miRNA misexpression is an important factor in human disease, including cancer. Consistent with this prediction, numerous studies have linked altered miRNA expression to cancer, both as diagnostic markers and as functional contributors. For pheochromocytoma, a prior study identified several miRNAs that were differentially expressed in malignant tumors (Meyer-Rochow et al. 2010). In particular, miR-483-5p was found to be overexpressed, and miR-15a and miR-16 were underexpressed in malignant as compared to benign pheochromocytomas (Meyer-Rochow et al. 2010). In this study, we further evaluated the miRNA expression changes that accompany malignancy in an independent, large pheochromocytoma tumor sample set, which includes both hereditary and sporadically occurring tumors. Moreover, we performed a comprehensive analysis of factors that may be associated with miRNA misexpression in pheochromocytoma tumor samples, including clinical characteristics, heredity, disease site, and germline mutation status. Finally, we evaluated the expression of miRNAs that are differentially expressed in malignant tumors in the serum of pheochromocytoma patients.

\section{Materials and methods}

\section{Tissue samples}

Pheochromocytoma samples were obtained at the time of surgery, snap frozen in liquid nitrogen, and stored at $-80^{\circ} \mathrm{C}$. Patient demographic, clinical, and pathologic information were collected under an Institutional Review Board (IRB)-approved protocol after written informed consent was obtained from patients. The patient and tumor characteristics of the samples used in this study are summarized in Table 1. Patients had genetic testing for mutations or large deletions in $R E T$, $V H L, S D H B, S D H C$, and $S D H D$. The diagnosis of NF1 was made clinically in one of the cases.

A diagnosis of malignant pheochromocytoma was made when there was clinical evidence of tumor at distant sites from the primary tumor location where chromaffin cells are not found or when there was gross local invasion.

Normal adrenal glands were procured from healthy organ donors under an IRB-approved protocol. Laser capture microdissection was used to isolate tissue from the medulla.

\section{RNA extraction and quality control}

Total RNA was extracted from frozen tissue as described (Patterson et al. 2011). RNA integrity and quality were confirmed using an Aligent 2100 Bioanalyzer (Agilent Technologies, Inc., Santa Clara, CA, USA). All RNA samples used for miRNA profiling had an RNA integrity number $>5$. All the normal adrenal medulla samples had an RNA integrity number $>5$.

\section{miRNA microarray expression profiling and data analysis}

A total of 50 microarrays were used to compare miRNA expression in benign and malignant pheochromocytomas as previously described (Patterson et al. 2011). Briefly, for each array, $300 \mathrm{ng}$ of total RNA (either tumor or reference) were labeled with $\mathrm{Cy} 3$ or Cy5 using the Array Labeling Kit (Exiqon, Vedbaek, Denmark). A pool of 21 normal adrenal medulla tissue

Table 1 Clinical characteristics for the samples used in this study

\begin{tabular}{|c|c|c|c|}
\hline & Total & Benign & Malignant \\
\hline Number of patients & 69 & 42 & 27 \\
\hline Age $\left(\right.$ years) ${ }^{\mathrm{a}}$ & $43.8 \pm 15.4(13-79)$ & $46.6 \pm 15.3(18-79)$ & $39.8 \pm 14.8(13-72)$ \\
\hline Gender (female/male) & $31 / 38$ & $22 / 20$ & $9 / 18$ \\
\hline Greatest tumor diameter $(\mathrm{cm})^{\mathrm{b}}$ & $5.0 \pm 3.0(1.3-15.0)$ & $4.7 \pm 2.5(1.3-13.0)$ & $5.4 \pm 3.9(1.7-15.0)$ \\
\hline \multicolumn{4}{|l|}{ Site } \\
\hline Adrenal & 47 & 40 & 7 \\
\hline Extra-adrenal & 22 & 2 & 20 \\
\hline Hereditary (\%) & 39.4 & 28.6 & 50.0 \\
\hline \multicolumn{4}{|l|}{ Positive mutation } \\
\hline$S D H B$ & 12 & 0 & 12 \\
\hline$R E T$ & 9 & 8 & 1 \\
\hline$V H L$ & 1 & 1 & 0 \\
\hline$N F 1$ & 1 & 1 & 0 \\
\hline
\end{tabular}

${ }^{\mathrm{a}}$ Mean \pm S.E.M. (range).

bean \pm s.D. (range). 
samples was used as a common reference on all arrays. Labeled RNA was hybridized to miRNA arrays (Exiqon miRCURY LNA miRNA arrays $\mathrm{v}$ 11.0). This array contains 7720 probes, 3300 of which represent 825 human miRNAs with four duplicate probes per miRNA. There are additional 1400 probes for miRNA in mouse or rat and 472 probes for miRNA in human, rat and human/mouse viruses. In addition, there are a variety of control and other probes: 1640 empty, 476 spike_control, 28 negative control, 12 U6snRNA, 60 hsa_SNORD, 1728 miRPlus, four 5SrRNA, and 240 Hy3. Dye swap was also performed as technical replicates. Fluorescence intensity was determined using an Axon GenePix 4000B scanner (Molecular Devices, Inc., Sunnydale, CA, USA) and GenePix Pro 6.0 Software (Molecular Devices, Inc., Sunnydale, CA, USA).

For data analysis, GenPix files were loaded into $\mathrm{R} /$ Bioconductor using the array package. Flagged spots were removed, and the remaining probes were used for normalization and subsequent analyses. The $\log _{2}$ of the intensities of $\mathrm{Cy} 3 / \mathrm{Cy} 5$ signals was calculated for each miRNA on every array (with no background subtraction) and normalized by print-tip loess normalization (Yang et al. 2002, Smyth 2004, Smyth et al. 2005). Because individual miRNAs were represented by four probes on the array, the median of normalized $\log _{2}$ ratio of the replicate probes (for those with more than two unflagged probes) was used as the expression value for the miRNA. The summarized $\log _{2}$ ratios for each experiment were then used for moderated $t$-statistics and $P$ value calculation using the limma package in $\mathrm{R} /$ Bioconductor, with adjustment for false discovery rate (FDR) using the Benjamini-Hochberg method (Smyth et al. 2005).

\section{Real-time quantitative RT-PCR analysis}

TaqMan real-time quantitative RT-PCR (qRT-PCR) was used to measure individual miRNAs in tumor samples. Specific miRNA primers (TaqMan MicroRNA Assay, PN 4427975; Applied Biosystems, Carlsbad, CA, USA) and TaqMan MicroRNA RT Kit (PN 4366596; Applied Biosystems) were used to synthesize single-stranded cDNA from $5 \mathrm{ng}$ total RNA. Then, $2 \mu \mathrm{l}$ cDNA was used as a template in a $10 \mu \mathrm{l}$ PCR reaction mix.

PCR amplification was performed using specific primers (TaqMan MicroRNA Assay; Applied Biosystems) and TaqMan Universal PCR Master Mix (PN 4324018; Applied Biosystems). The 7900HT Fast RealTime PCR System (Applied Biosystems) was used for quantification of the PCR product. All PCR reactions were run in triplicate and cDNA synthesized without reverse transcriptase enzyme (RNA only) and no cDNA templates were used for negative controls. The following TaqMan MicroRNA Assays used in this study were obtained from Applied Biosystems: hsa-miR-483-5p (ID 2338), hsa-miR-101 (ID 2253), hsa-miR-513a-5p (ID 2090), hsa-miR-183 (ID 2269), has-miR-16 (ID), mmu-miR-710 (ID 1645), and U6 (ID 1973). miRNA expression level was expressed either as the cycle threshold $\left(C_{\mathrm{t}}\right)$ or the difference between the $C_{\mathrm{t}}$ of the miRNA of interest and the $C_{\mathrm{t}}$ of $\mathrm{U} 6\left(\Delta C_{\mathrm{t}}\right)$. The average U6 $C_{\mathrm{t}}$ value for the normal adrenal medulla samples was 21 with a coefficient of variance of $5.8 \%$ across all these samples.

\section{Serum miRNA measurements}

RNA was isolated from $0.4 \mathrm{ml}$ patient serum using Trizol LS (Invitrogen) according to the manufacturer's instructions. After chloroform extraction, 1.5 volumes of $100 \%$ ethanol were added to the aqueous phase. RNA was purified from this mixture using the Qiagen RNeasy Kit (Qiagen) according to the manufacturer's protocol. RNA was eluted in $50 \mu \mathrm{l}$ water.

For miRNA quantification, a fixed volume of RNA was used (either 1 or $2 \mu \mathrm{l}$ ) for cDNA synthesis. RT and TaqMan reactions were performed as described above. Reactions without RNA template were used as a control and the value obtained was considered the background level of miRNA expression. miR-16 expression was also measured as this miRNA has been suggested to be highly and stably expressed in human serum (Mitchell et al. 2008). As a normalization strategy to correct for assay variation, miRNA expression was also measured in the human adrenocortical carcinoma cell line, H295R (ATCC, Rockville, MD, USA; $5 \mu \mathrm{g}$ RNA input). cDNA reactions lacking

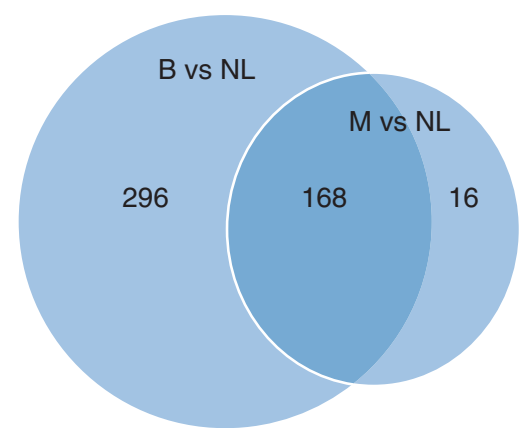

Figure 1 Number of differentially expressed miRNAs in pheochromocytoma tumors (benign, $\mathrm{B}$; malignant, $\mathrm{M}$ ) as compared to normal adrenal medulla tissue $(\mathrm{NL})$ identified by microarray profiling. Differentially expressed miRNAs were defined using an FDR-adjusted $P$ value $\leq 0.05$. 
Table 2 The differentially expressed miRNAs between benign and malignant pheochromocytomas identified by microarray analysis

\begin{tabular}{|c|c|c|c|c|}
\hline Probe name & $\begin{array}{c}\text { Mean } \log _{2} \\
\text { (malignant/benign) }\end{array}$ & $\begin{array}{l}\text { Fold difference } \\
\text { (malignant/benign) }\end{array}$ & Raw $P$ value & Adjusted $P$ value $^{a}$ \\
\hline hsa-miRPlus-E1246 b & 0.686 & 1.609 & 0.0017 & 0.010 \\
\hline hsa-miRPlus-E1030 & 0.932 & 1.908 & 0.0039 & 0.021 \\
\hline hsa-miR-183 & 0.813 & 1.757 & 0.0047 & 0.025 \\
\hline hsa-miR-483-5p & 0.965 & 1.952 & 0.0088 & 0.042 \\
\hline hsa-miR-513a-5p & 0.400 & 1.320 & 0.0130 & 0.057 \\
\hline hsa-miRPlus-E1106 & 0.198 & 1.147 & 0.0150 & 0.066 \\
\hline mmu-miR-710 & 0.449 & 1.365 & 0.0160 & 0.067 \\
\hline hsa-miR-101 & -0.873 & 0.546 & 0.0190 & 0.078 \\
\hline
\end{tabular}

${ }^{a}$ Adjusted for false discovery rate using the Benjamini-Hochberg method.

${ }^{\mathrm{b}}$ Exiqon proprietary miRNA.

RNA template were used as the negative control. miRNA expression was expressed as $C_{\mathrm{t}}$.

\section{Statistical analysis}

For the qRT-PCR data, the Mann-Whitney $U$ test was used to determine statistical significance for all the comparisons. A $P$ value of $<0.05$ was considered statistically significant. All calculations were performed using StatView 4.57 Statistical Software (Abacus Concepts, Inc., Cary, NC, USA). Receiver operating characteristic (ROC) curve generation and area under the ROC curve (AUC) calculations were performed using SPSS version 16.0 (Chicago, IL, USA).

\section{Results}

\section{Identification of differentially expressed miRNAs}

To assess the miRNA expression differences in pheochromocytoma samples, we performed whole genome microarray profiling of 50 clinical samples (eight malignant and 42 benign). Two arrays were excluded due to poor quality (two malignant). We first examined the differences between miRNA expression in benign and malignant tumors compared to the pooled normal adrenal medulla samples (Fig. 1). We found that 464 and 184 miRNAs were differentially expressed in benign and malignant pheochromocytomas respectively as compared to normal. Interestingly, the majority of differentially expressed miRNAs in malignant pheochromocytomas as compared to normal were also misexpressed in benign tumors, which included sporadic and a variety of familial disease (Table 1). Overall, there were few significant differences in the expression levels of miRNAs between benign and malignant tumors.

Given that all of the tumor samples were compared with a common reference (pooled normal adrenal medulla) on the arrays, we were able to directly compare the expression differences in benign and malignant tumors. Using an FDR-adjusted $P$ value cutoff of $<0.1$, there were eight miRNAs differentially expressed between malignant and benign pheochromocytomas (Table 2).

\section{Unsupervised cluster analysis}

Unsupervised hierarchical clustering of the 50 most variably expressed miRNAs was performed to determine if there was grouping of the pheochromocytoma samples according to clinical or pathologic features (Fig. 2). Although there was some minimal structure, there was no clear clustering of samples based on any of the variates.

\section{QRT-PCR analysis of differentially expressed micoRNAs}

Independent miRNA measurements by qRT-PCR were performed on a cohort that included the same samples used for microarray analysis and additional malignant and benign tumor samples (62 total: 25 malignant, 36 benign, and one pooled normal adrenal medulla). Of the eight most significantly dysregulated miRNAs (Table 2), we directly tested the expression of five miRNAs (miR-483-5p, miR-101, miR-513, miR-183, and miR-710) using qRT-PCR. miR-483-5p, miR-101, and miR-183 had significant differential expression between benign and malignant samples (Table 3 and Fig. $3, P=0.0068, P=0.0001$, and $P<0.0001$ respectively). miR-710 was lowly expressed in all of the samples tested and was therefore excluded from further analysis. For miR-513-5p, there was no significant difference in expression between benign and malignant samples (Table 3 and data not shown, $P=0.7603$ ).

To begin to understand the basis for these differences in pheochromocytomas, we examined the relationship between miRNA expression and specific clinical and pathologic factors. There were no 


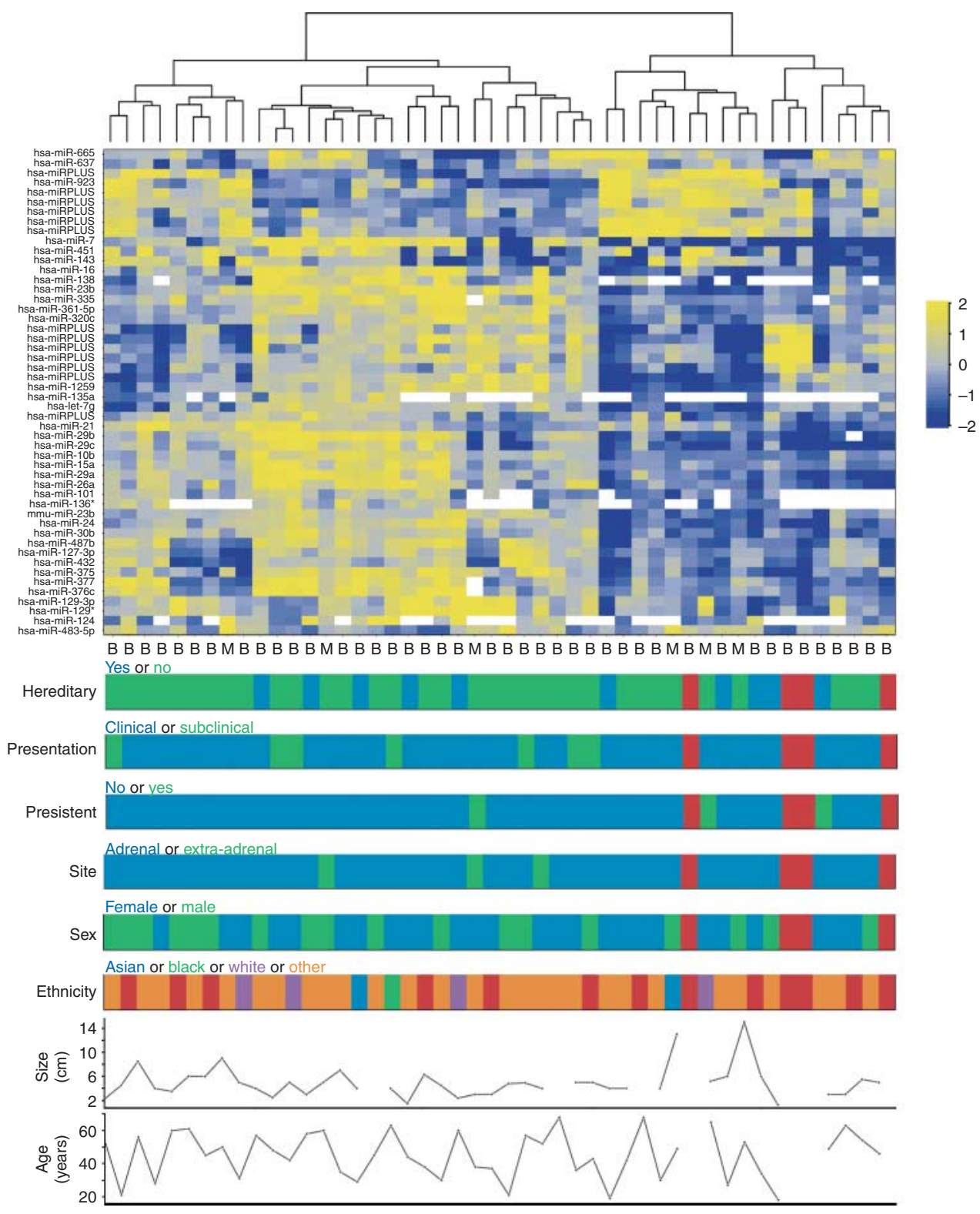

Figure 2 Heatmap of the 50 most variably expressed miRNAs. Unsupervised hierarchical clustering was performed using Euclidian distance as the similarity metric. Expression variability was defined by greatest median absolute deviation across all samples. Each row represents an miRNA and each column an individual tumor sample. 'miR-Plus' miRNAs are proprietary to Exiqon. Tumor type is indicated below the heatmap as either benign (B) or malignant (M). Demographic and clinical data are plotted below the heatmap (red indicates data not available).

significant miRNA expression differences when samples were compared based on age, gender, ethnicity, or tumor size. However, samples from patients with hereditary pheochromocytomas tended to have higher expression of miR-183 than those with sporadic pheochromocytomas (Fig. 4A, $P=0.05$ ). In addition, when samples with succinate dehydrogenase subunit B $(S D H B)$ mutations were compared with all non-SDHB mutation samples, we found that miR-101 and miR-183 were significantly different (Fig. 4B and C, $P=0.0015$ and $P=0.0002$ respectively). Interestingly, for this comparison miR-483-5p expression did not differ; however, when only the malignant samples were compared, miR-483-5p expression was significantly lower in $S D H B$ mutation positive tumors vs all other genotypes (Fig. 4D, $P=0.0267$ ).

Although the majority of pheochromocytomas are adrenal, $\sim 10 \%$ are extra-adrenal. Extra-adrenal 
Table 3 The miRNA expression differences and diagnostic accuracy in benign and malignant tumors

\begin{tabular}{llrrr}
\hline & $\begin{array}{c}\text { Direction of } \\
\text { change in } \\
\text { malignant }\end{array}$ & $\begin{array}{c}\text { Benign vs } \\
\text { malignant } \\
\boldsymbol{P} \text { value }\end{array}$ & AUC & $\begin{array}{r}\boldsymbol{P} \text { value } \\
\text { for AUC }\end{array}$ \\
\hline miR-483-5p & Upregulated & 0.0068 & 0.70 & 0.007 \\
miR-101 & Upregulated & 0.0001 & 0.78 & $<0.0001$ \\
miR-183 & Upregulated & $<0.0001$ & 0.82 & $<0.0001$ \\
miR-513-5p & NA & 0.7603 & ND & ND \\
\hline
\end{tabular}

NA, not applicable; ND, no data. Area under the receiver operating characteristic (ROC) curve.

ander the null hypothesis that the true area $=0.5$.

tumors have a higher likelihood of being malignant. Consistent with this, there was a significant difference in the expression of the malignancy-associated miRNAs, miR-101 and miR-183 (Fig. 4E and F, $P=0.0017$ and $P=0.0191$ respectively) between adrenal and extra-adrenal tumors.

\section{Diagnostic accuracy of differentially expressed miRNAs}

Based on the identification of several miRNAs that were upregulated in malignant tumors (Fig. 3), we next assessed if any were accurate diagnostic markers. Specifically, ROC curves were plotted for each miRNA based on the qRT-PCR values (Fig. 5A). All of the miRNAs had high and significant AUC values (Table 3), suggesting their usefulness in molecular diagnostics.

Since carriers of $S D H B$ mutations are likely to have malignant pheochromocytoma (Timmers et al. 2009), we further assessed the ability of miRNA expression to be a marker of malignancy in non-SDHB tumors. In this subanalysis, we again found that miR-101, miR183, and miR-483-5p were good markers, with AUC values of $0.76,0.77$, and 0.77 respectively (Fig. 5B, all $P<0.01)$.

\section{Diagnostic miRNA expression in serum}

A number of recent studies have indicated that tumorderived miRNAs are detectable in body fluids and expression level can be used to predict tumor type. Given these findings, we were interested in examining the expression of the candidate diagnostic miRNAs (miR-101, miR-183, and miR-483-5p) in pheochromocytoma patient serum. A total of 16 serum samples were analyzed (nine from patients with benign tumors and seven from patients with malignant tumors). QRTPCR measurement showed that the miRNAs were detectable in the serum, and although the expression level was low, they were above background in all of the
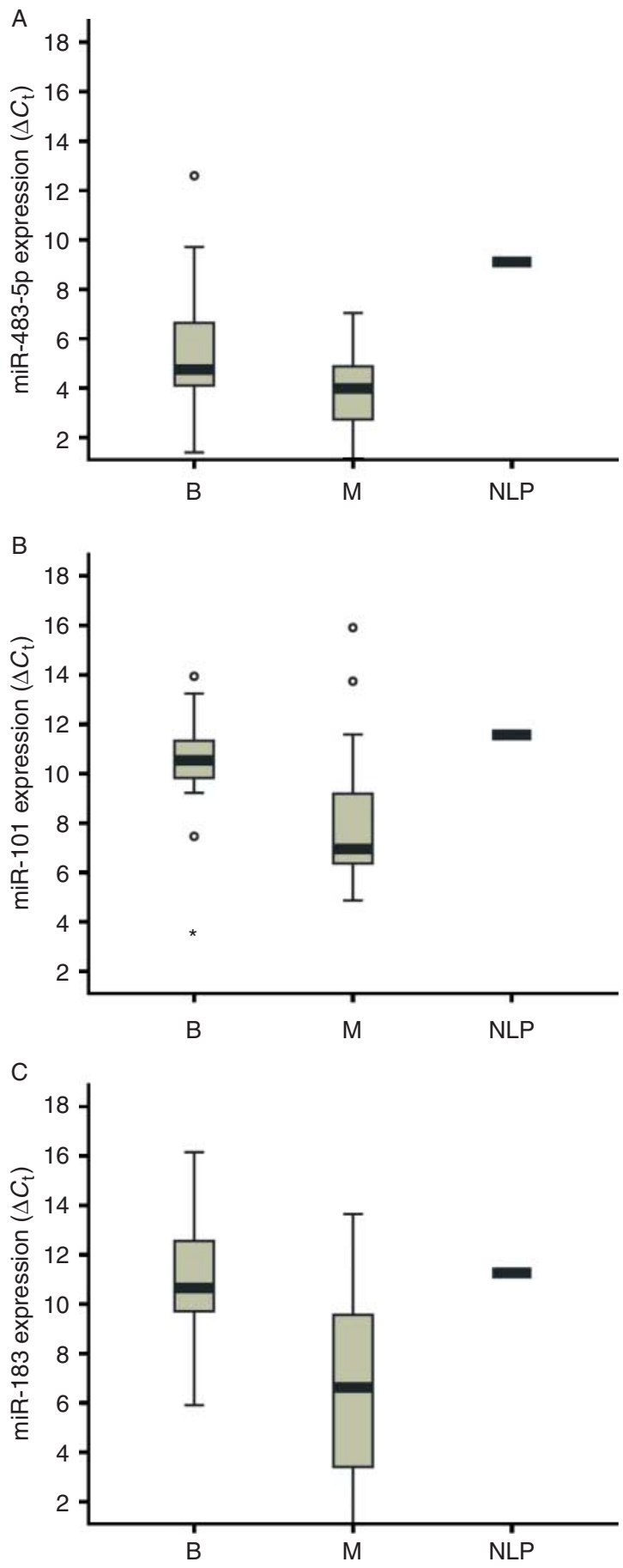

Figure 3 miRNA expression in benign and malignant tumors and normal adrenal medulla tissue (pool of 21 samples). The box plots represent (A) miRNA-483-5p, (B) miR-101, and (C) miR-183 expression as defined as the $\Delta C_{t}$, as described in the Materials and Methods. A lower $\Delta C_{t}$ indicates higher miRNA expression level. In these graphs, potential outliers are indicated by either open circles (outlier) or asterisks (extreme outlier). B, benign; M, malignant; NLP, normal. 

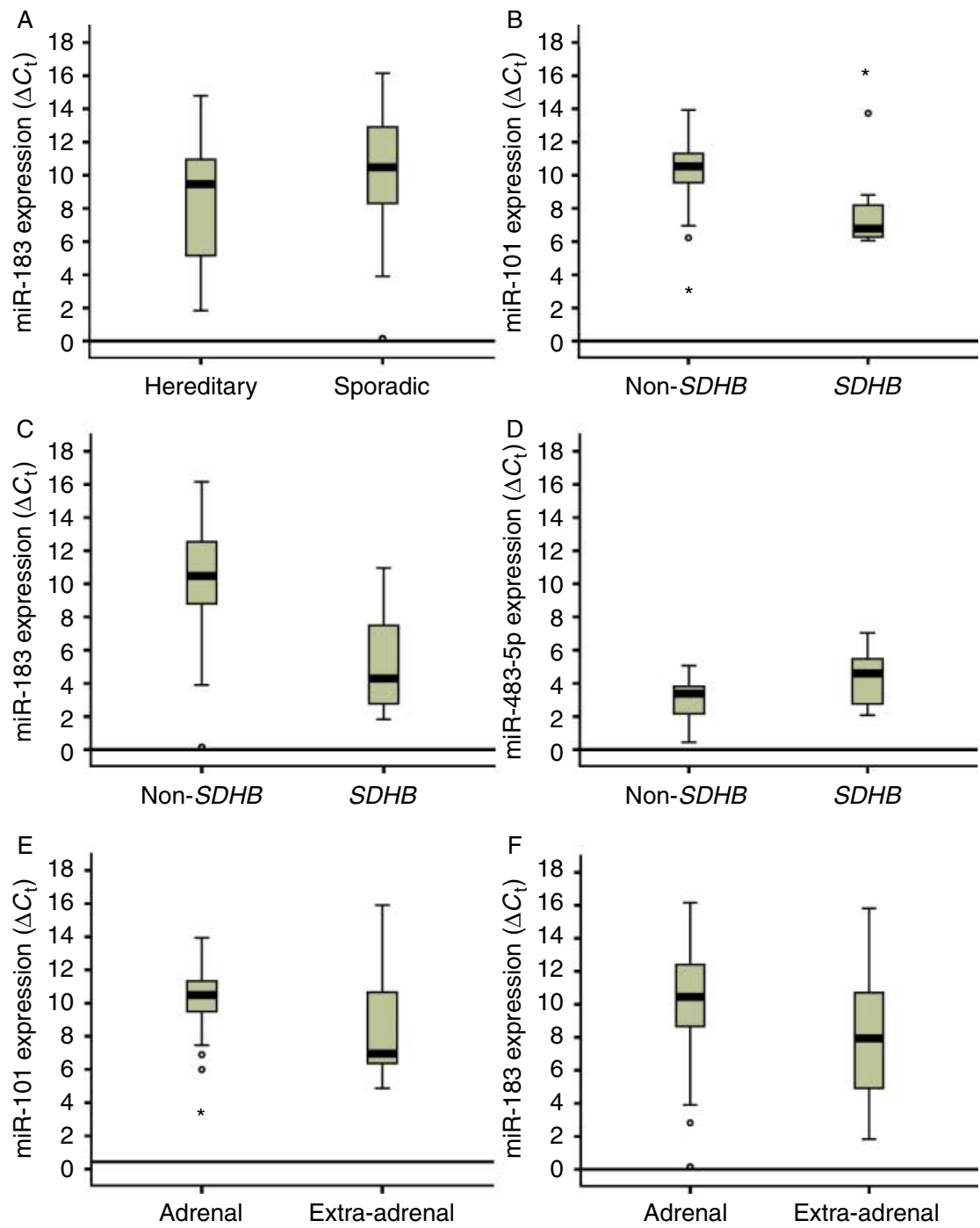

Figure 4 miRNA expression differences in samples categorized by various clinical factors. Expression is defined as the $\Delta C_{t}$, as described in the Materials and Methods. A lower $\Delta C_{\mathrm{t}}$ indicates higher miRNA expression level. miRNA expression differences were found in the comparison of (A) hereditary vs sporadic, (B, C and D) SDHB vs non-SDHB, (E and F) and adrenal vs extra-adrenal tumors. In these plots, potential outliers are indicated by either open circles (outlier) or asterisks (extreme outlier).

samples (Fig. 6). However, there was no difference in serum miR-483-5p, miR-101, or miR-183 expression in patients with benign compared to those with malignant tumors (Fig. 6, $P=0.2235, P=0.4273$, $P=0.3146$ respectively). The same result was obtained when the expression for each miRNA was normalized to miR-16 (i.e. $\Delta C_{\mathrm{t}}$; data not shown).

\section{Discussion}

In this study, miRNA profiling of pheochromocytomas was used to identify miRNAs that differ in expression between benign and malignant tumors. We found significant misexpression of miR-483-5p, miR-101, and miR-183 in malignant pheochromocytomas as compared to their benign counterparts. Moreover, these miRNAs had relatively high diagnostic accuracies (AUC values of $0.694,0.783$, and 0.783 respectively) and were detectable in pheochromocytoma patient serum samples. In addition, we were able to identify specific clinical factors that were associated with this miRNA misexpression.

To address the need for diagnostic markers for malignant pheochromocytomas, a number of studies have focused on identifying the molecular changes that accompany malignancy. In terms of miRNA misexpression, a previous study performed in an independent sample set found that miR-483-5p was overexpressed 


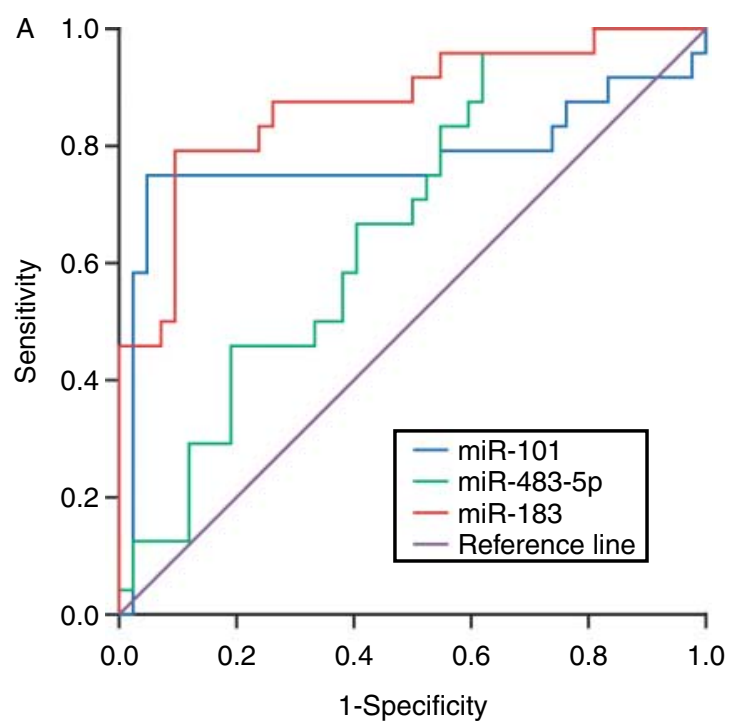

B

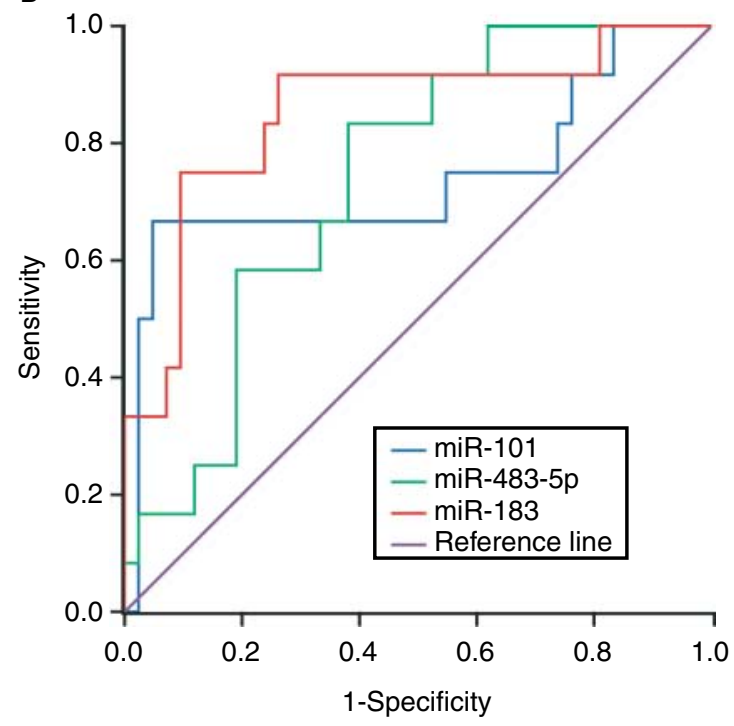

Figure 5 Receiver operating characteristic $(\mathrm{ROC})$ curves for the differentially expressed miRNAs. The curves were generated from the $\Delta C_{\mathrm{t}}$ measurements for $(\mathrm{A})$ all tumor samples or $(\mathrm{B})$ only non-SDHB tumor samples.

and miR-15a and miR-16 were underexpressed in malignant pheochromocytomas (Meyer-Rochow et al. 2010). In addition, Tombol et al. (2010) used formalinfixed paraffin-embedded samples to identify the upregulation of miR-1225-3p in sporadic recurrent pheochromocytomas as compared to sporadic benign tumors (Tombol et al. 2010). Both of these studies indicate that miRNAs are misexpressed in malignant pheochromocytomas and are an important class of potential diagnostic/prognostic markers and therapeutic targets, which warrant further investigation.
This study expanded on these findings in a large cohort and confirmed that miR-483-5p may be useful as an adjunct diagnostic marker for distinguishing benign from malignant pheochromocytomas. In addition, our work identified two additional miRNAs, miR-101 and miR-183, that are also misexpressed in malignant pheochromocytomas.

Several clinical and pathologic factors and germline mutations are associated with malignant pheochromocytoma (Zarnegar et al. 2006, Grogan et al. 2011). To begin to dissect the basis for miRNA expression differences in pheochromocytomas, we examined the relationship between the differentially expressed miRNAs and clinical factors associated with malignancy. For example, extra-adrenal tumors are more often malignant. Indeed, we found that miR-101 and miR-183, which were differentially expressed in malignant samples, were also expressed differently depending on the tumor site (adrenal vs extra-adrenal). However, one caveat to the interpretation of this finding is that the vast majority of the extra-adrenal samples in our cohort were malignant (Table 1).

$S D H B$ encodes a subunit of the mitochondrial complex II, and mutations in $S D H B$ lead to hereditary PGLs and occur at a high rate in malignant pheochromocytomas (Blank et al. 2010). In addition, several studies have shown an association between $S D H B$ mutations and shorter overall survival in patients with malignant pheochromocytoma and PGL

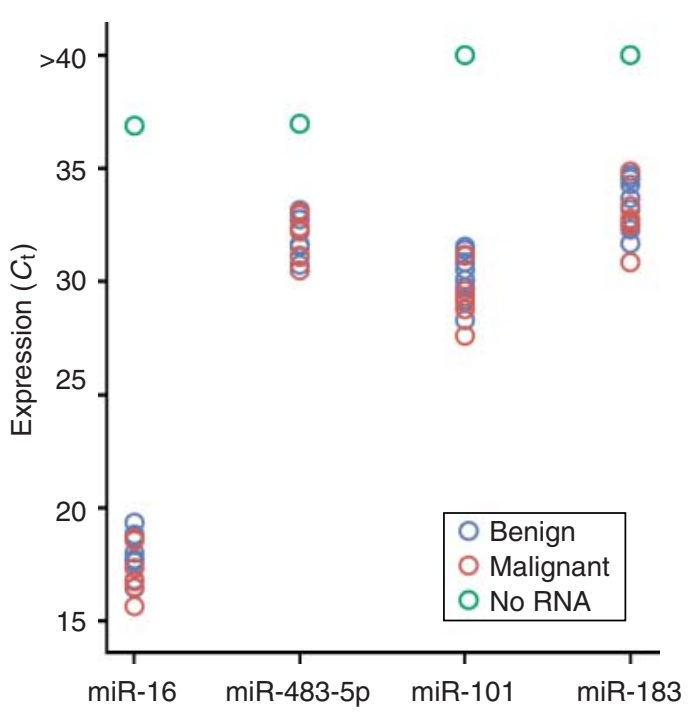

Figure 6 Expression level of miRNAs in patient serum. The scatter plot shows the miRNA expression as the $C_{\mathrm{t}}$ measured by qRT-PCR for each sample. Serum samples from patients with benign tumors (blue), patients with malignant tumors (red), or the no RNA template control (green) were included in this analysis. A lower $C_{\mathrm{t}}$ indicates higher miRNA expression level. 
(Gimenez-Roqueplo et al. 2003, Benn et al. 2006, Amar et al. 2007, Plouin et al. 2010). We found significant expression differences in miR-101 and miR183 when samples with $S D H B$ mutations were compared with all non-SDHB genotypes (RET, $V H L$, $N F 1$, and sporadic). This difference is likely due to the increased rate of malignancy in $S D H B$ samples. However, amongst just the malignant samples there were significant expression differences only for miR483-5p for this comparison, with malignant samples having lower expression.

There is much promise of using miRNA expression analysis for molecular diagnostics and prognostication of cancer (Ferracin et al. 2010). Here, we identified several miRNAs with high AUC values. Not only were these markers accurate for characterizing pheochromocytomas in all samples, but were also able to predict tumor type in non-SDHB tumors. Therefore, clinically we envision using $S D H B$ mutational analysis first, and subsequently, for those non-SDHB cases, using miRNA expression analysis to assess risk of malignancy. In addition, we were able to detect miR-483-5p, miR-183, and miR-101 expression above background in serum samples of patients with pheochromocytoma. Additional studies will be necessary to assess the clinical utility of testing serum miRNA expression level as a diagnostic strategy for pheochromocytoma.

Little is known regarding the molecular alterations that lead to malignant pheochromocytoma. We speculate that the miRNAs identified in this study may shed some light on this subject. Specifically, all of the miRNAs that differed in expression between benign and malignant pheochromocytomas have been linked to different types of cancers. Interestingly, a recent study in adrenocortical carcinoma revealed a growthpromoting role for miR-483-5p in vitro (Caramuta et al. 2011). In addition, miR-183 has been shown to regulate EGRl and PTEN, important tumor-suppressor genes (Sarver et al. 2010, Hannafon et al. 2011).

In summary, we have identified miRNAs that are differentially expressed in benign vs malignant pheochromocytomas. Furthermore, our work indicates that miR-483-5p, miR-101, and miR-183 are good diagnostic markers for distinguishing malignant from benign pheochromocytomas and that these miRNAs are present in serum samples from patients with pheochromocytomas.

\section{Declaration of interest}

The authors declare that there is no conflict of interest that could be perceived as prejudicing the impartiality of the research reported.

\section{Funding}

This research was supported by the Intramural Research Program of the NIH, National Cancer Institute, Center for Cancer Research.

\section{References}

Amar L, Baudin E, Burnichon N, Peyrard S, Silvera S, Bertherat J, Bertagna X, Schlumberger M, Jeunemaitre X, Gimenez-Roqueplo AP et al. 2007 Succinate dehydrogenase B gene mutations predict survival in patients with malignant pheochromocytomas or paragangliomas. Journal of Clinical Endocrinology and Metabolism 92 3822-3828. (doi:10.1210/jc.2007-0709)

Benn DE, Gimenez-Roqueplo AP, Reilly JR, Bertherat J, Burgess J, Byth K, Croxson M, Dahia PL, Elston M, Gimm O et al. 2006 Clinical presentation and penetrance of pheochromocytoma/paraganglioma syndromes. Journal of Clinical Endocrinology and Metabolism 91 827-836. (doi:10.1210/jc.2005-1862)

Blank A, Schmitt AM, Korpershoek E, van Nederveen F, Rudolph T, Weber N, Strebel RT, de Krijger R, Komminoth P \& Perren A 2010 SDHB loss predicts malignancy in pheochromocytomas/sympathethic paragangliomas, but not through hypoxia signalling. Endocrine-Related Cancer 17 919-928. (doi:10.1677/ERC-090316)

Caramuta S, Ozata DM, Velazquez-Fernandez D, Akcakaya P, Xie H, Hoog A, Zedenius J, Backdahl M, Larsson C \& Lui WO 2011 The role of microRNA deregulation in the pathogenesis of adrenocortical carcinoma. EndocrineRelated Cancer 18 643-655. (doi:10.1530/ERC-11-0082)

Comino-Mendez I, Gracia-Aznarez FJ, Schiavi F, Landa I, Leandro-Garcia LJ, Leton R, Honrado E, Ramos-Medina R, Caronia D, Pita G et al. 2011 Exome sequencing identifies MAX mutations as a cause of hereditary pheochromocytoma. Nature Genetics 43 663-667. (doi:10.1038/ng.861)

Ferracin M, Veronese A \& Negrini M 2010 Micromarkers: miRNAs in cancer diagnosis and prognosis. Expert Review of Molecular Diagnostics 10 297-308. (doi:10. 1586/erm.10.11)

Gimenez-Roqueplo AP, Favier J, Rustin P, Rieubland C, Crespin M, Nau V, Khau Van Kien P, Corvol P, Plouin PF \& Jeunemaitre X 2003 Mutations in the $S D H B$ gene are associated with extra-adrenal and/or malignant phaeochromocytomas. Cancer Research 63 5615-5621.

Grogan RH, Mitmaker EJ \& Duh QY 2011 Changing paradigms in the treatment of malignant pheochromocytoma. Cancer Control 18 104-112.

Hannafon BN, Sebastiani P, de Las Morenas A, Lu J \& Rosenberg CL 2011 Expression of microRNA and their gene targets are dysregulated in preinvasive breast cancer. Breast Cancer Research 13 R24. (doi:10.1186/bcr2839)

Meyer-Rochow GY, Jackson NE, Conaglen JV, Whittle DE, Kunnimalaiyaan M, Chen H, Westin G, Sandgren J, Stalberg P, Khanafshar E et al. 2010 MicroRNA profiling 
of benign and malignant pheochromocytomas identifies novel diagnostic and therapeutic targets. EndocrineRelated Cancer 17 835-846. (doi:10.1677/ERC-10-0142)

Mitchell PS, Parkin RK, Kroh EM, Fritz BR, Wyman SK, Pogosova-Agadjanyan EL, Peterson A, Noteboom J, O'Briant KC, Allen A et al. 2008 Circulating microRNAs as stable blood-based markers for cancer detection. PNAS 105 10513-10518. (doi:10.1073/pnas.0804549105)

Neumann HP, Sullivan M, Winter A, Malinoc A, Hoffmann MM, Boedeker CC, Bertz H, Walz MK, Moeller LC, Schmid KW et al. 2011 Germline mutations of the TMEM127 gene in patients with paraganglioma of head and neck and extraadrenal abdominal sites. Journal of Clinical Endocrinology and Metabolism 96 E1279-E1282. (doi:10.1210/jc.2011-0114)

Patterson EE, Holloway AK, Weng J, Fojo T \& Kebebew E 2011 MicroRNA profiling of adrenocortical tumors reveals miR-483 as a marker of malignancy. Cancer 117 1630-1639. (doi:10.1002/cncr.25724)

Plouin PF, Amar L \& Lepoutre C 2010 Phaeochromocytomas and functional paragangliomas: clinical management. Best Practice \& Research. Clinical Endocrinology \& Metabolism 24 933-941. (doi:10.1016/j.beem.2010.10.002)

Qin Y, Yao L, King EE, Buddavarapu K, Lenci RE, Chocron ES, Lechleiter JD, Sass M, Aronin N, Schiavi F et al. 2010 Germline mutations in TMEM127 confer susceptibility to pheochromocytoma. Nature Genetics $\mathbf{4 2}$ 229-233. (doi:10.1038/ng.533)

Sarver AL, Li L \& Subramanian S 2010 MicroRNA miR-183 functions as an oncogene by targeting the transcription factor EGR1 and promoting tumor cell migration. Cancer Research 70 9570-9580. (doi:10.1158/0008-5472.CAN10-2074)

Smyth GK 2004 Linear models and empirical bayes methods for assessing differential expression in microarray experiments. Statistical Applications in Genetics and Molecular Biology 3 (Article 3) 1-26. (doi:10.2202/1544-6115.1027)
Smyth GK, Michaud J \& Scott HS 2005 Use of within-array replicate spots for assessing differential expression in microarray experiments. Bioinformatics 21 2067-2075. (doi:10.1093/bioinformatics/bti270)

Timmers HJ, Gimenez-Roqueplo AP, Mannelli M \& Pacak K 2009 Clinical aspects of SDHx-related pheochromocytoma and paraganglioma. Endocrine-Related Cancer 16 391-400. (doi:10.1677/ERC-08-0284)

Tombol Z, Eder K, Kovacs A, Szabo PM, Kulka J, Liko I, Zalatnai A, Racz G, Toth M, Patocs A et al. 2010 MicroRNA expression profiling in benign (sporadic and hereditary) and recurring adrenal pheochromocytomas. Modern Pathology 23 1583-1595. (doi:10.1038/modpathol.2010.164)

Yang YH, Dudoit S, Luu P, Lin DM, Peng V, Ngai J \& Speed TP 2002 Normalization for cDNA microarray data: a robust composite method addressing single and multiple slide systematic variation. Nucleic Acids Research 30 e15. (doi:10.1093/nar/30.4.e15)

Yao L, Schiavi F, Cascon A, Qin Y, Inglada-Perez L, King EE, Toledo RA, Ercolino T, Rapizzi E, Ricketts CJ et al. 2010 Spectrum and prevalence of FP/TMEM127 gene mutations in pheochromocytomas and paragangliomas. Journal of the American Medical Association 304 2611-2619. (doi:10.1001/jama.2010. 1830)

Zarnegar R, Kebebew E, Duh QY \& Clark OH 2006 Malignant pheochromocytoma. Surgical Oncology Clinics of North America 15 555-571. (doi:10.1016/j.soc. 2006.05.009)

Received in final form 20 December 2011

Accepted 10 January 2012

Made available online as an Accepted Preprint 12 January 2012 